\title{
El Liderazgo Emocional Y Su Repercusión En Las Instituciones Educativas Españolas
}

\author{
Vanesa Román Costela (Doctorando) \\ Departament of Didactic \& School Organization, \\ University of Granada, Spain \\ Juan Ruiz Lucena (Inspector de Educación y Doctor en \\ Derecho) \\ Departament of Didactic \& School Organization, \\ University of Granada, Spain
}

doi: 10.19044/esj.2016.v12n28p96 URL:http://dx.doi.org/10.19044/esj.2016.v12n28p96

\begin{abstract}
This article presents a theoretical review based in one of the emerging new leadership models: emotional leadership; likewise, the impact of exercising this style of leadership in Spanish educational institutions. These effects are in the areas of education, the physical and mental health of members of the educational community, improved interpersonal relationships, avoidance of stress and job anxiety. Finally, it will conclude with the importance of including Emotional Intelligence in training programs for headmasters through coaching, to emphasize the importance of exercising an emotional leadership style as a means of improving the organization and quality of Spanish schools.
\end{abstract}

Keywords: Emotional leadership, educational institutions, training of school managers, coaching

\section{Resumen}

En este artículo se realiza una revisión teórica fundamentada de uno de los nuevos modelos de liderazgo emergente: el liderazgo emocional; así como, de las repercusiones de ejercer este estilo de liderazgo en las instituciones educativas españolas. Estas repercusiones giran en torno al ámbito educativo, a la salud física y mental de los miembros de la comunidad educativa, la mejora de las relaciones interpersonales, evitar el estrés y la ansiedad laboral. Por último, se concluirá con la importancia de incluir la Inteligencia emocional en los programas de formación de los directivos escolares a través del "coaching”, para recalcar la importancia de 
ejercer un estilo de liderazgo emocional como medio de mejora de la organización y de la calidad de los centros educativos españoles.

Palabras clave: Liderazgo emocional, instituciones educativas, formación de directivos escolares, coaching

\section{Introducción}

Existen distintas clasificaciones de los modelos de liderazgo que han ido evolucionando en función de las nuevas demandas sociales y que están estrechamente relacionadas con los llamados estilos de liderar y que son, atendiendo a las metáforas o visiones en la literatura científica de Lorenzo (2001) las siguientes: Centrado en principios (Covey, 1995), Intuitivo (Le Saget, 1997), Transcultural (Kreitner y Kinicki, 1996), Global (Kreitner y Kinicki, 1996), Líder como entrenador (Durcam y Oates, 1994), Estratégico (Ansoff, 1997), Visionario (Nanus, 1994), De liberación (Noer, 1997), Instructivo (Greenfield, 1987).

Esta clasificación fue completada y ampliada por el mismo Lorenzo (2005) y más tarde adaptada por Cáceres Reche (2007). Puede verse en la tabla 1 del Anexo.

Según las conclusiones extraídas de las clasificaciones de ambos profesores y sus respectivos estudios; podemos decir que los modelos de liderazgo son muy variados en función de las características determinantes de los estilos de liderar. Tanto es así, que parece fundamental considerar el modelo de liderazgo atendiendo a las características peculiares del contexto donde se está llevando a cabo.

Traducido al ámbito educativo, existen tantos estilos de liderazgo como centros educativos. Esta afirmación es afín a la teoría de la contingencia o situacional tal y como afirmaron Tannenbaum y Schmidt (1958), Fiedler (1967), House (1977), Hersey y Blanchard, (1987) y Lorenzo (2005). Si bien, los más conocidos estilos de liderazgo clásicos podrían englobarse en cinco modelos: el situacional, el transformacional, el pedagógico, el relacional y el trascendente.

Por otro lado, cabe considerar otros modelos de liderazgo emergentes como son el liderazgo sostenible y el liderazgo emocional. El primero de ellos pretende que todas las mejoras realizadas en los centros puedan ser perdurables y sostenibles para garantizar la mejora de la calidad y del rendimiento escolar. Mientras que el liderazgo emocional se basa en el establecimiento de relaciones adecuadas entre las personas que forman un equipo de trabajo. A este último modelo de liderazgo emocional dedicamos las siguientes páginas de este artículo. 


\section{Hacia un nuevo modelo de liderazgo: el liderazgo emocional.}

Goleman (2012) sentó las bases para este nuevo modelo de liderazgo diciendo: "Los factores que mejor discriminan de entre un grupo de personas igualmente inteligentes, a quiénes mostrarán una mayor capacidad de liderazgo, no son el CI (cociente intelectual) ni las habilidades técnicas, sino las relaciones con la IE” (p.18).

Además, Goleman (2016) nos da una visión de las cualidades que debe poseer un líder "resonante” que según Mayer (2006) son: "Aquella persona capaz de influir positivamente en las emociones del grupo, gestionando y canalizando la información emocional que recibe de manera que se produzca un efecto contagio que acabe creando un óptimo clima emocional donde trabajar" (p.2).

Otra investigadora en este ámbito, es Agüero (2009), que va un poco más allá y propone lo siguiente:

La próxima generación de líderes debe estar en capacidad de manejar presiones de gran intensidad, formar recursos humanos de alta calidad integral y desarrollar comunidades corporativas ejemplares por su solidez estructural, misión y su visión de compromiso en organizaciones de aprendizaje, con directivos preparados....con sólida arquitectura organizacional. (p.53)

El liderazgo del futuro es o debe ser emocional, compartido, contextualizado y transformacional. Como dicen Álvarez, M., Díaz, V., Estefanía, J.L. et al (2007):

Entendemos el estilo de un director/a escolar del futuro como un/a profesional de la educación que ejerce un liderazgo compartido, situacional, emocional y transformacional, capaz de implicar a sus colaboradores en un proyecto de futuro pensado en la satisfacción de las expectativas tanto de sus colaboradores como de las personas interesadas en la educación: alumnado y familias. (p.89)

La idea que proponen estos autores llega a ser la clave del nuevo modelo de liderazgo emocional que se propone a través de nuestra investigación. Si entendemos el liderazgo de forma compartida, esto quiere decir que implica relaciones interpersonales entre distintos “colaboradores” como aparece en la cita anterior, y traducido a nuestro contexto educativo actual se refiere a todos y cada uno de los miembros de la comunidad educativa. Por tanto, si queremos implicar a toda la comunidad educativa: ¿No serían necesarias una serie de habilidades sociales científicamente demostradas, que poseen aquellas personas que tienen un alto índice de Inteligencia Emocional? La respuesta es afirmativa; y, deberíamos añadir, que no sólo serían necesarias sino que resultan, a todas luces, totalmente imprescindibles. 
De que mejor forma un director escolar puede motivar, persuadir y animar a su equipo directivo y al claustro de maestros/as en la realización de proyectos de mejora e innovación educativos; que a través de la negociación, la persuasión, el "gana-gana” (técnica de resolución de conflictos en la que las dos partes sacan un beneficio al llegar a un acuerdo) o la motivación, entre otros. No se nos ocurre mejor forma de hacerlo, y en este sentido se desenvuelven aquellos líderes considerados emocionalmente inteligentes.

\section{El liderazgo emocional y su repercusión en las instituciones educativas}

Tras una revisión bibliográfica se exponen, a continuación, algunas aportaciones de distintos autores sobre la importancia de un liderazgo emocional en la mejora de la organización y de la calidad de los centros educativos.

La educación del s.XXI está basada, según el informe realizado por Delors para la UNESCO (2013), en cuatro pilares fundamentales a lo largo de la vida de una persona: aprender a saber, aprender a hacer, aprender a ser y aprender a vivir juntos; en este sentido, trabaja la Inteligencia Emocional a la que nos referimos, más concretamente, se refieren a los dos últimos y como diría Hargreaves (2008):

Aprender a ser, aprender a vivir juntos y aprender a vivir de forma sostenible, constituyen retos emocionales, morales y espirituales, y no sólo cognitivos e intelectuales. Toda enseñanza y todo aprendizaje son prácticas emocionales, para bien o para mal, porque así se quiera o por negligencia. (p.45)

Además, una de las cualidades que todo líder que ejerza un liderazgo sostenible debería poseer es la capacidad para "Crear las condiciones emocionales para el aprendizaje” (Hargreaves y Fink, 2008, p.48).

Agüero Rossi (2009), profundizó en dicha materia llevando a cabo una investigación la sobre el liderazgo creativo emocional para optimizar la calidad del proceso educativo en la Universidad Pedagógica Experimental Libertador en Barquisimeto (Venezuela), llegando a afirmar lo siguiente: "El modelo propuesto no solamente va a la parte educativa sino que reconoce al liderazgo, a la creatividad y a la inteligencia emocional como pilares fundamentales en la formación del ser humano, como líder integral y transformador".(p.69)

Con respecto a las relaciones que genera este modelo, Agüero Rossi (2009) propuso que: "La relación de influencia que ocurre entre los líderes (elementos) y sus seguidores debe ser abordada como objeto mediante el cual las dos partes pretenden llegar a cambios (emociones) y resultados reales (creatividad) que reflejen los propósitos que comparten”. (p.69)

En definitiva, podemos afirmar que el liderazgo emocional tendrá repercusiones significativas en las organizaciones educativas en las que sea 
implementado; ya que, influye en la salud física y mental de los miembros de la comunidad educativa y en la mejora de las relaciones interpersonales de sus miembros creando lazos de grupo como veremos más adelante.

Además, cabe señalar uno de los datos extraídos en la investigación realizada por Teixidó (2008) sobre las competencias de los directivos escolares, puesto que hace alusión a que algunas de las competencias que los directores entrevistados señalaron como más difícil de conseguir y desempeñar fueron: "El Control emocional, seguida del Liderazgo, la Fortaleza Interior y la Adaptación al cambio" (p.8). Es por ello, que una vez más, se pone de manifiesto la importancia de ampliar la formación de los futuros directores escolares en inteligencia emocional.

\section{Repercusiones de la IE en el ámbito educativo}

De Pelakis, C., \& Pereira, J. (2010) se pronunciaron, respecto las repercusión en las organizaciones del liderazgo con inteligencia emocional, afirmando que: "Un estilo de vida emocionalmente inteligente garantiza una vida más larga y más sana, gracias a una red amistosa de personas que brindan apoyo, a una toma de decisiones más prudente, a unas relaciones familiares más compasivas, a una disposición generosa y al dominio del estrés”. (p.44)

Cada vez mayor número de investigaciones refutan esta teoría y avalan las ventajas de la inteligencia emocional como una competencia del directivo escolar que debería ser transversal a todas las demás, e incluso, impregnar todas las actuaciones del líder. Cabría destacar algunas de estas investigaciones en torno a la mejora de la salud física y mental, mejora de las relaciones interpersonales y antídoto contra la aparición del estrés laboral y la ansiedad. A continuación, explicaremos brevemente algunas de estas aportaciones.

En resumen, Goleman muy recientemente (2015) nos aportaba que: "La inteligencia emocional se trasmite a través de una organización como la electricidad a través de unos cables. Para ser más específico el estado de ánimo del líder es, literalmente, contagioso, se propaga con facilidad y de manera inexorable por toda la empresa”. (p.73)

En nuestro caso la "empresa" se trata del centro educativo y las instituciones públicas.

\section{Respecto a la salud física y mental de los miembros de la comunidad educativa}

Resultan asombrosas algunas de las afirmaciones de Goleman (1995) el cual nos advierte sobre las repercusiones de la IE sobre la salud física y mental de las personas, por ende de los miembros de la comunidad educativa: 
La toxicidad de las emociones negativas crónicas es equiparable a la ocasionada por el tabaquismo. Es por ello por lo que ayudar a que la gente domine mejor estas emociones comporta un beneficio médico potencial tan importante como lograr que un fumador empedernido abandone su hábito. (p.114)

En la sociedad actual, donde a la escuela y a los maestros/as cada vez les son exigidas mayor número de funciones (muchas de ellas meros trámites burocráticos) y donde existen ciertos riesgos de salud para el profesorado propios de los ambientes escolares donde se desenvuelven; como el alto nivel de ruido (provocado por el mal aislamiento de las aulas, una ratio elevada, mala sonoridad y ecos), el cual obliga a los profesores a forzar sus cuerdas vocales, provocan riesgos para su salud física y mental que son muy difíciles de erradicar debido a la falta de medios materiales para ello.

Sin embargo, estos riesgos de la salud se verían mermados si se dotará a los docentes de las herramientas emocionales adecuadas, y esto parece factible a través de un liderazgo emocional, democrático, compartido, transformacional y pedagógico.

En las investigaciones en el campo de la medicina realizadas por Goleman (1995) en las que colaboró con algunos neurocientíficos, llegaron a conclusiones relevantes del tipo: "Existe una ingente cantidad de investigaciones que subrayan la conexión existente entre el cerebro emocional y el sistema inmunológico". (p.114)

Recordemos que el sistema inmunológico central es el encargado de protegernos de diversas enfermedades a través de generación de las defensas contra los microorganismos nocivos para la salud.

En este sentido, y aprovechando que la infancia es una puerta abierta a la oportunidad gracias a la plasticidad cerebral, Goleman (1995) propone una misión extra para las escuelas, se trata de enseñar a ser y enseñar a respetar; en definitiva, se trata de repensar la escuela:

El proceso de alfabetización emocional impone una carga adicional a la escuela (...) Una difícil tarea que exige dos cambios esenciales: que los maestros vayan más allá de la misión que tradicionalmente se les ha encomendado y que los miembros de la comunidad se comprometan más con el mundo escolar. (p.116)

Consideremos tras lo expuesto que ha pasado más de una década desde que Daniel Goleman dio fama mundial a la teoría de la inteligencia emocional; las escuelas en la actualidad no ven la alfabetización emocional como una carga más, sino todo lo contrario porque se han dado cuenta que han mejorado los rendimientos a través de la formación del profesorado en esta temática. Y aunque podemos decir que la IE está cada vez siendo más y mejor integrada en el currículo del alumnado y en la formación permanente 
del profesorado, todavía no se ha avanzado lo suficiente en la inclusión de la misma en la formación de los futuros directores escolares.

\section{Respecto a la mejora de las relaciones interpersonales.}

Son indudables los beneficios que la IE aporta a las relaciones que se establecen en las organizaciones, por ende en los centros educativos.

La alfabetización emocional lleva implícitas una serie de habilidades que mejoran no sólo la competencia emocional sino también la social, y aunque como hemos señalado anteriormente este hecho está demostrado sobre la población infantil y juvenil, como afirman Extremera y Berrocal (2004):

Estudios anglosajones han encontrado datos empíricos entre la IE y las relaciones interpersonales (...) En países como Estados Unidos por en el que utilizaron medidas de habilidad como MSCEIT, se han hallado evidencias sobre la relación entre IE y la calidad de las relaciones sociales (Lopes, Salovey y Straus ,2003). Los estudiantes que puntúan alto en IE mostraron mayor satisfacción en las relaciones con sus amigos e interacciones más positivas.

Cabría destacar entre las ventajas de una alfabetización emocional, las relacionadas con “Dirigir las relaciones” que, según Goleman (1995), son las siguientes:

Mayor capacidad de analizar y comprender las relaciones, mejora en la capacidad de resolver conflictos y negociar desacuerdos, mejora en la solución de los problemas de relación, mayor afirmatividad y destreza en la comunicación, mayor popularidad y sociabilidad, amistad y compromiso con los compañeros, mayor atractivo social, más preocupación y consideración hacia los demás, más sociables y armoniosos en los grupos, más participativos, cooperadores y solidarios y más democráticos en el trato con los demás. (p.174)

Si además de todo lo anterior, tenemos en cuenta que el desarrollo de la Inteligencia Emocional implica la mejora de habilidades sociales, la capacidad de empatía, la asertividad, el optimismo,...parece evidente que estamos en el buen camino y no deberíamos abandonar esta formación ni en las escuelas desde los niveles iniciales ni en las etapas adultas ni, por supuesto, en la formación de líderes escolares.

En definitiva y para concluir, nos parecen interesantes las reflexiones sobre inteligencia emocional de Jiménez Muniáin (2008) que dice:

Hay una mayor correlación del nivel de éxito personal y social de la persona con su grado de inteligencia emocional que con su nivel de inteligencia cognitiva. La inteligencia emocional es una capacidad clave para toda persona la hora de interrelacionarse eficazmente. Debe formar parte del carácter profundo de la persona e ir unida a la tenacidad y 
capacidad del esfuerzo. No se trata de meras simpatía o empatía, sino de fuerza, exigencia, optimismo y perseverancia para ser creíble y eficaz. (p.59)

\section{Respecto al estrés y la ansiedad laboral.}

Como ya hemos ido adelantando, la sociedad del siglo XXI está mediatiza por un ritmo de vida vertiginoso y acelerado, que también se ve reflejado en nuestras escuelas. Este ritmo frenético al que nos vemos sometidos a diario, por horarios impuestos, por exigencias laborales y por ser una de las características de las sociedades creadas en torno al consumismo, nos provoca tensiones, ansiedades y estrés que tienen graves repercusiones para nuestro organismo.

Relacionado con el estrés y la ansiedad laboral, aparece también el "burnout" (síndrome del quemado) el cual se está convirtiendo en una de las mayores plagas de los sistemas educativos actuales. Su origen es multifactorial; si bien, podrían señalarse aspectos como el desprestigio social de los maestros y profesores, la crisis económica y los consecuentes recortes salariales, la poca incentivación de las administraciones por programas innovadores y por la investigación educativa en general, entre otros

Daniel Goleman (2015) afirmaba, de forma reciente en su libro “¿Cómo ser un líder?”, lo siguiente: “La inteligencia emocional de un líder crea una cierta cultura o un cierto ambiente de trabajo. Según mostró la investigación, los altos niveles de inteligencia emocional crean ambientes en los que florece el intercambio de información, la confianza, el aprendizaje y una saludable asunción de riesgos. Bajos niveles de inteligencia emocional crean ambientes en los que abundan el miedo y la ansiedad”. (p.72)

En este sentido, trabajan los programas de formación en IE aplicados al profesorado y cabría señalar las conclusiones extraídas de las investigaciones llevadas a cabo por Extremera, Berrocal y Durán (2003) en el ámbito de la IE y la aparición del "burnout”:

Los hallazgos de este estudio proporcionan evidencias sobre ciertos factores emocionales y cognitivos relacionados con la aparición de desajuste emocional y burnout que deben ser tenidos en cuenta en futuros programas dirigidos a la prevención, formación y entrenamiento en el control del estrés laboral del profesorado. (p.264)

Una vez argumentadas las ventajas para la salud física y psicológica de los individuos con adecuado desarrollo emocional, parece conveniente señalar a continuación cómo se traducirían estas ventajas en la inclusión de la IE en los programas de formación novel y permanente de los directivos escolares. 


\section{Importancia de la inclusión de la IE en los programas de formación de directivos escolares.}

Como diría Senge (1993): "Las organizaciones que destacarán verdaderamente en el futuro serán las que descubran como suscitar el compromiso y la capacidad de las personas para aprender en todos los niveles de una organización”. (p.26)

Este compromiso implica un cambio necesario en las organizaciones escolares, y cabría señalar que muchos profesores son reacios a los cambios pues se sienten cómodos en lo que ya conocen y no quieren asumir ciertos riesgos. Es por ello, que para que estos programas de entrenamiento emocional sean implantados entre los profesores, y más concretamente, entre los futuros directivos escolares sería útil disponer de una motivación extrínseca.

Senge (1993) se refirió a esta motivación como las “palancas para el cambio" y las describió como: “Acciones que tienen el efecto de hacer avanzar la práctica, al modificar el comportamiento de una organización y de los individuos que están en ella”. (p.28)

Una vez hallada la determinación para cambiar a mejor, a través de programas de formación en IE, no sólo por parte del profesorado y de los directivos sino también de las administraciones educativas (ya que son las encargadas de regular estos programas de formación) podremos identificar las ventajas que conllevaría para la calidad de los centros y el rendimiento escolar de los alumnos/as.

Atendiendo a las aportaciones de Vargas Jiménez (2007): "La inteligencia emocional se compone de cinco aspectos, de los cuáles se vinculan con el liderazgo: las relaciones interpersonales, las relaciones intrapersonales y la empatía”. (p.86)

Siguiendo con lo anterior, los líderes (en nuestro caso directivos escolares) que posean un grado elevado de IE, deberían ser capaces de: manejar relaciones tensas que puedan generarse en el contexto educativo y tratar con personas “difíciles”, serán capaces de conocer sus propias emociones y exteriorizarlas o controlarlas en los momentos adecuados, de sentir lo que le sucede al interlocutor, saber escuchar y ser comprendido; y por último, serán capaces de motivar a los demás y de automotivarse. En definitiva, los directivos escolares que sean inteligentes emocionales destacarán por tener un adecuado desarrollo de la inteligencia interpersonal y la intrapersonal; así como d la empatía y la capacidad para motivar equipos y automotivarse en la consecución de unos objetivos comunes.

\section{Conclusion}

Como afirma Vargas Jiménez (2007): 
Se podría determinar que un buen liderazgo significa una adecuada inteligencia emocional o una adecuada inteligencia emocional significa poseer habilidades que se requieren para mantener y prolongar el liderazgo. (...) Los directores escolares deben conocer y procurar alcanzar el máximo provecho de sus potencialidades, aplicando para ello la inteligencia emocional con liderazgo. (p.86)

Por tanto, parece obvia la importancia de ajustar los programas de formación de directivos escolares a las necesidades reales de los centros, incluyendo entre las competencias exigidas para el desempeño de su cargo la competencia emocional.

Es por ello que compartimos con Gairín (2004) que: "Nuestro problema real es promover el paso de unos directivos ignorados e ignorantes a reconocidos y formados (...). La solución pasa por clarificar la encrucijada de elementos que inciden en la función directiva y, por ende, en la formación correspondiente”. (p.22)

Por otro lado, siguiendo a Sarramona, Vázquez y Ucar (1991) deberemos tener en cuenta que las necesidades de formación de los directivos escolares integran elementos como: habilidades y conocimientos vinculados a las tareas, aptitudes y actitudes vinculadas a la relación social y al desarrollo personal. Y esto nos hace preguntamos: ¿Qué mejor programa de formación que aquel que incluya la inteligencia emocional con el objetivo de desarrollar estas habilidades, aptitudes y actitudes?

Es por ello, que desde diferentes esferas de la sociedad (ámbito empresarial, ámbito educativo e incluso en el ámbito personal y social) se están haciendo verdaderos esfuerzos por llevar a cabo programas de formación que potencien y desarrollen el entrenamiento emocional.

Cabría destacar la existencia de una corriente de entrenamiento emocional iniciada en el ámbito empresarial llamada "coaching” que se define del siguiente modo, según la psicóloga Ortiz de Zarate (2010):

El coaching es una disciplina que nació en Estados Unidos con el objetivo de incrementar el rendimiento individual de los profesionales del deporte. Desde entonces han transcurrido 30 años y estas lecciones se han extrapolado en los más diversos campos de la actividad humana. La estructura empresarial ha sabido hacer suya esta enseñanza y cada vez son más los profesionales de diferentes sectores que recurren a esta alternativa para mejorar su desempeño. (p.56)

Este proceso de entrenamiento está íntimamente relacionado con la inteligencia emocional, ya que el “coach" o entrenador debe estar en posesión de una serie de habilidades socio-emocionales que son las que le van a permitir motivar a una persona o a un equipo en la consecución de objetivos propuestos. Podríamos encontrar, por tanto, cierto paralelismo 
entre la figura del “coach” y la del líder (o en el ámbito educativo el directivo escolar).

Para reforzar esta idea utilizaremos las palabras de Ortiz de Zarate (2010) que dicen lo siguiente: "Hoy en día, grandes organizaciones e instituciones educativas de primera línea reconocen las importantes aportaciones del coaching al desarrollo del liderazgo en todo el mundo”. (p.59)

En definitiva, la importancia de desarrollar las dimensiones englobadas en la IE (las competencias emocionales) parece obvia y ampliamente justificada a lo largo de este artículo; y como valoración final nos gustaría añadir que un adecuado desarrollo a nivel emocional nos permitirá ser personas inteligentes emocionales y alcanzar nuestros propósitos, haciendo sentirnos útiles en la sociedad en la que nos ha tocado vivir, incluso llegando a cotas nunca soñadas de éxito y felicidad en nuestras vidas.

\section{References:}

1. Agüero Rossi, C. (2010). Aproximación teórica a un modelo de liderazgo creativo emocional en el contexto universitario. (p. 51-70).

2. Cáceres, Ma . P. (2007). El liderazgo estudiantil en la Universidad de Granada desde una perspectiva de género. Mención Europea. Universidad de Granada, Tesis doctoral.

3. De Pelakis, C., \& Pereira, J. (2010). La inteligencia emocional del líder como estrategia de cambio en las organizaciones. CICAG, 2 (Vol. 1) Pag.36-45. Consultado el 20 de Septiembre, 2016, de http://publicaciones.urbe.edu/index.php/cicag/article/view/505/1262

4. Delors, J. (2013). Los cuatro pilares de la educación. Galileo, (23).

5. Extremera, N., Fernández Berrocal, P., \& Durán, A. (2003). Inteligencia emocional y burnout en profesores. Encuentros en Psicología Social, 1(5), 260-265.

6. Gairín Sallán, J. (2004b). La dirección de directivos: ¿Una encrucijada con solución? Organización y Gestión Educativa, 11(2), 22-27.

7. Goleman, D., Boyatzis, R., \& McKee, A. (2016). El líder resonante crea más. Barcelona: De bolsillo

8. Goleman, D. (2015). Cómo ser un líder. Barcelona: Ediciones B. Grupo Zeta.

9. Goleman, D. (2012). Inteligencia Emocional. Barcelona: Kairós.

10. Goleman, D. (1995). La práctica de la inteligencia emocional. Barcelona: Kairós. 
11. Hargreaves, A. \& Fink, D. (2008). El liderazgo sostenible. Siete principios para el liderazgo en centros educativos innovadores. Madrid: Ediciones Morata.

12. Jiménez Muniáin, S. (2008a). Cuestión de confianza. Más allá de la inteligencia emocional. ( $2^{\mathrm{a}}$ ed.). Madrid: ESIC.

13. Lorenzo Delgado, M. (1998). Las visiones actuales del liderazgo en las instituciones educativas. En Lorenzo Delgado, M., Ortega Carrillo, J.A e Sola Martínez, T. (coords.): Enfoques en la organización y dirección de instituciones educativas formales y no formales. Granada: Grupo Editorial Universitario/Asociación para el Desarrollo de la Comunidad Educativa en España, 27-44.

14. Lorenzo Delgado, M. L. (2005). El liderazgo en las organizaciones educativas: revisión y perspectivas actuales. Revista española de pedagogía, 367-388.

15. Mayer, J. (2006). Unidad de Conocimiento: La inteligencia emocional (p. 1-6). Fundación para la motivación de los recursos humanos. Recuperado de http://www.factorhuma.org/attachments_secure/article/8301/IE_cast. pdf

16. Ortiz de Zarate, M. (2010). Psicología y Coaching: marco general, las diferentes escuelas. Capital Humano, 243, 56-58.

17. Extremera Pacheco, N. \& Fernández-Berrocal, P. (2004). El papel de la inteligencia emocional en el alumnado: evidencias empíricas. Revista electrónica de investigación educativa, 6(2). Recuperado de http://redie.uabc.mx/vol6no2/contenidoextremera.html el 20 de Septiembre de 2016.

18. Sarramona, J., Vázquez, G., \& Ucar, J. (1991). Evaluación de la educación no formal. (X Seminario Interuniveristario de Teoría y Educación). Oviedo: Universidad de Oviedo.

19. Senge (1993). La quinta disciplina. Barcelona: Garnica.

20. Teixidó, J., Aramendi, P., Bernal, J. L., Fernández, M., \& Rodríguez, J. (2008a). Competencias profesionales de los directivos escolares. Identificación y desarrollo. Organizaciones educativas al servicio de la sociedad Actas del X CIOIE, 1.

21. Teixidó Saballs, J. (2008b). Competencias profesionales inherentes al ejercicio de la dirección escolar. Identificación y desarrollo. Presentado en la Ponencia de las XVII Jornadas de Equipos Directivos de Educación Infantil y Primaria. La Coruña.

22. Vargas Jiménez, I. (2007). El ejercicio del liderazgo y su relación con la inteligencia emocional en la administración educativa. Educare, $X$ (1), 79-88. 
Tabla 1: Tipos de liderazgo

\begin{tabular}{|c|c|}
\hline Tipos de liderazgo & Conceptos \\
\hline $\begin{array}{c}\text { Ético } \\
\text { (García y Dolan,1997) }\end{array}$ & $\begin{array}{l}\text { Una organización, especialmente educativa, } \\
\text { implica por naturaleza confrontación de valores. }\end{array}$ \\
\hline $\begin{array}{c}\text { Carismático } \\
\text { (Conger, 1991) } \\
\end{array}$ & $\begin{array}{l}\text { El liderazgo no sólo encarna la visión del } \\
\text { centro sino su transmisión con un estilo propio. }\end{array}$ \\
\hline $\begin{array}{l}\text { Liderazgo con vocación de servicio } \\
\text { (Autry, 2003; Zohar, 2001) }\end{array}$ & $\begin{array}{c}\text { El liderazgo entendido como una función } \\
\text { de servicio a la institución y sus miembros. }\end{array}$ \\
\hline $\begin{array}{l}\text { Liderazgo resonante } \\
\text { (Goleman, 2002) }\end{array}$ & $\begin{array}{c}\text { Dinamiza en base a } \\
\text { la inteligencia emocional. }\end{array}$ \\
\hline $\begin{array}{c}\text { E-liderazgo } \\
\text { (Quinn Mills, 2002) }\end{array}$ & $\begin{array}{c}\text { Es el liderazgo afín a las ciber-organizaciones } \\
\text { y a las comunidades virtuales. }\end{array}$ \\
\hline $\begin{array}{c}\text { Liderazgo lateral } \\
\text { (Fisher y Sharp, 1999) }\end{array}$ & $\begin{array}{c}\text { El liderazgo basado en ciertas habilidades } \\
\text { personales y en el desarrollo de las mismas en los } \\
\text { miembros del grupo. }\end{array}$ \\
\hline $\begin{array}{l}\text { Liderazgo basado en los resultados } \\
\text { (Ulrich, 2000) }\end{array}$ & $\begin{array}{c}\text { Aquel que dinamiza la organización } \\
\text { en función de los resultados o productos. }\end{array}$ \\
\hline $\begin{array}{l}\text { Liderazgo sin Límites } \\
\text { ( Heifetz y Linsky, 2003) }\end{array}$ & $\begin{array}{l}\text { El liderazgo que no le importa que surja el } \\
\text { conflicto, el desafío de creencias arraigadas } \\
\text { ni el reto de ver las cosas de otra manera. }\end{array}$ \\
\hline $\begin{array}{c}\text { Liderazgo emocional } \\
\text { (Fernández y otros, 2001) }\end{array}$ & $\begin{array}{c}\text { Es una metáfora similar a la de Goleman. } \\
\text { Es un director de emociones. }\end{array}$ \\
\hline $\begin{array}{l}\text { Liderazgo creativo } \\
\text { (Dilts, 1998) }\end{array}$ & $\begin{array}{c}\text { Trabaja la creatividad y con creatividad } \\
\text { preocupándose de la innovación institucional. }\end{array}$ \\
\hline $\begin{array}{l}\text { Liderazgo estratégico } \\
\text { (Bou, 2004) }\end{array}$ & $\begin{array}{l}\text { La innovación es el elemento } \\
\text { clave de la dirección escolar actual. }\end{array}$ \\
\hline $\begin{array}{l}\text { El líder narcisista } \\
\text { (Maccoby, 2004) }\end{array}$ & $\begin{array}{c}\text { Destaca por su ansia de ser } \\
\text { el centro de atención de toda la organización. }\end{array}$ \\
\hline $\begin{array}{l}\text { Liderazgo clarividente } \\
\text { (Sharma, 2003) }\end{array}$ & En él predomina la visión de futuro. \\
\hline
\end{tabular}

Nota: Tomada de Cáceres Reche (2007). 